\title{
Development of Memory Structures for Homographs Using Pathfinder Network Representations
}

\author{
Francisco Nievas \\ University of Almería
}

\author{
Fernando Justicia \\ University of Granada
}

\begin{abstract}
Some studies with children have shown that there is no semantic priming at short stimulus onset asynchrony (SOA) in lexical decision and naming tasks for homographs. The predictions of spreading activation theories might explain this missing effect. There may be differences in children's and adults' memory structures. We have explored this hypothesis. The development of memory structure representations for homographs was measured by a Pathfinder algorithm. In Experiment 1, the three dependent variables were: the number of links in the network, closeness measures (C), and distances between nodes. Results revealed developmental differences in network structure representations in adults and children. In Experiment 2, results revealed that these differences were not due to the cohort effect. In Experiment 3, the relationship between associative strength, as measured by associative norms, and distances, as measured by Pathfinder algorithm, was explored. The results of these three experiments and empirical research from semantic priming experiments show that these differences in memory structure representations could be one of the sources of the missing semantic priming effect in children.

Keywords: Pathfinder, memory structure representations, semantic priming, cohort effect
\end{abstract}

Algunos estudios con niños han mostrado que no existe facilitación semántica, con intervalos de tiempo cortos, en tareas de decisión léxica y en nombrar en homógrafos. Las predicciones de las teorías de difusión de la activación podrían explicar la ausencia de este efecto. Podría haber diferencias en las estructuras de memoria entre niños y adultos. Nosotros hemos explorado esta hipótesis. El desarrollo de las representaciones de estructuras de memoria para homógrafos se midió por el algoritmo Pathfinder. En el primer experimento, las tres variables dependientes fueron: el número de conexiones en la estructura, las medidas $\mathrm{C}$ y las distancias entre nodos. Los resultados revelaron diferencias evolutivas en las representaciones de las estructuras de red entre niños y adultos. En el experimento 2, los resultados mostraron que estas diferencias no eran debidas al efecto de cohorte. En el experimento 3, se exploró la relación entre la fuerza asociativa, medida por las normas de asociación, y las distancias, como medida del algoritmo Pathfinder. Los resultados de estos tres experimentos y la investigación empírica de los experimentos de facilitación semántica muestran que estas diferencias en las representaciones de estructuras de memoria podrían ser una de las fuentes para explicar la ausencia de facilitación semántica en niños.

Palabras clave: Pathfinder, representaciones de estructuras de memoria, facilitación semántica, efecto cohorte

We thank Pilar Sánchez, Luis Fuentes, and Roger Schvaneveldt for their help with this paper.

Correspondence concerning this article should be addessed to Francisco Nievas Cazorla, Departamento de Psicología Evolutiva y de la Educación, Universidad de Almería, La Cañada de San Urbano, 04120 Almería (Spain). E-mail: fnievas@ual.es 
In a word recognition paradigm (Besner \& Humphreys, 1991), stimuli such as lines and curves are presented visually, and participants must contact their meanings to understand them. This access is a phase of the reading processes, in which information is assumed to undergo a series of stages such as sublexical, lexical, sentence, and text (Vega, Carreiras, Gutiérrez-Calvo, \& Alonso-Quecuty, 1990). Word recognition occurs at the lexical level, where, in order to understand a word, individuals must first access the representations of its meaning, grammar, and phonological representations (Taft, 1991).

The semantic priming effect is important in word recognition (Meyer \& Schvaneveldt, 1971; for a review, see Neely, 1991). When a word is preceded by another, related word, this facilitates recognition of the original word, as opposed to when an unrelated word is used. That is, if butter is followed by bread, recognition of bread is faster than if bread is presented after nurse.

Much research has been carried out on the differences between children and adults in semantic priming (Nievas, 1999a, 1999b; Schvaneveldt, Ackerman, \& Semlear, 1977; Schwantes, 1981; Schwantes, Boesl, \& Ritz, 1980; Simpson \& Foster, 1986; Simpson \& Lorsbach, 1983; Stanovich, West, \& Feeman, 1981; West \& Stanovich, 1978). The most important finding is that semantic priming is higher in children than in adults (Schwantes et al., 1980). These researchers presented sentences as context before the target word. Subsequent to reading a sentence, there was an interstimulus interval (ISI) of 500 or $1000 \mathrm{~ms}$ after which participants had to make a lexical decision about the target word. Sentence length varied from one to eight words. Results showed that, with increasing sentence length, semantic priming differences between adults and children also increased.

But this result is not consistent. For example, Simpson, Krueger, Kang, and Elofson (1994), using a sentence context with long ISI, did not find this effect. That is, children's semantic priming was not greater than that of adults. However, whereas Simpson et al. (1994) used homographic words, Schwantes et al. (1980) used nonhomographic words. This might explain these discrepant results, because homographic words have specific properties (e.g., more than one meaning).

Simpson and Foster's (1986) study provides more evidence against children's higher semantic priming. In this investigation, a single, prime homograph was employed as a word context in a naming task. Stimulus onset asynchrony (SOA, that is, interval between the prime and target onset) varied from 150 to $750 \mathrm{~ms}$. The most intriguing result was that semantic priming was not higher in children than in adults; instead, at short SOA (less $250 \mathrm{~ms}$ ), there was no semantic priming in children, whereas it was present in adults (Frost \& Bentin, 1992; Nievas, Cañas, \& Bajo, 1994; Simpson \& Burgess, 1985). These results were replicated by Nievas (1999a, 1999b) in a lexical-decision task with homograph words in children (younger than 14 years old), finding no semantic priming at short SOA. Therefore, results are relatively consistent.

Can word recognition theories explain this? Coming from word recognition theories, spreading activation theories (Anderson, 1976, 1983; Collins \& Loftus, 1975; Collins \& Quillian, 1969; MacKay ${ }^{1}$, 1987, 1990; Neely, 1977; Posner \& Snyder, 1975) might explain more data at short SOA (Neely, 1991). For example, Collins and Loftus (1975) assumed that semantic knowledge is organized as a complex network of associated concepts. In the normal semantic network, concepts sharing many common attributes are more strongly associated than those that share fewer attributes. Specific attributes of a concept serve a dual purpose: (a) They provide the means for grouping concepts into categories, and (b) they differentiate the exemplars that make up a given category. According to this network model of semantic memory, each component of a spoken utterance activates the associated semantic concepts in a neural network. The model states that concepts thus activated in a semantic network facilitate spreading activation to other related concepts. Semantic units remain activated for a short period of time, after which activation either decays or is inhibited (e.g., Neely, 1977; Nievas \& Marí-Beffa, 2002).

In the standard lexical-decision task in which a prime word is presented first, participants should attend the prime, which is followed by a target word. Participants decide whether or not the target word has a meaning, pressing a key on the computer keyboard. According to semantic priming theories, when a prime word is presented, then the corresponding node is activated. This process spreads to connected nodes, so that when the target word is presented, its node has already been activated to some extent by the prime word node. Recognition is faster because the amount of activation needed to reach the recognition threshold is lower for the target word node. Activation decreases as interconnected nodes are further apart in the network.

Nievas (1999b) proposed some hypotheses to explain why priming is absent in children. ANOVAs may not capture priming effects in children because their reaction times are more variable than those of adults. Younger people's high variability has also been found in the heart and in other physiological systems (Goldberger, Rigney, Mietus, Antman, \& Greenwald, 1988; Goldberger, Rigney, \& West, 1990; Goldberger \& West, 1987; West \& Goldberger, 1987).

\footnotetext{
1 In this theory, activation does not spread but there is an analogous process that behaves in the same way. This process is called priming.
} 
Another explanation of the missing semantic priming in children is that they may have higher word-recognition thresholds, so that prime word activation does not occur. Note that the prime word was exhibited for $100 \mathrm{~ms}$ in Nievas' studies (1999a; 1999b). Another hypothesis is based on children's and adults' differences in concept networks. For adults, the prime word node and the target word node may be directly connected, whereas for children, they may be connected via some intermediate nodes. Therefore, when a prime word node spreads activation, the target word node does not receive the activation because of these intermediate nodes and because activation spreading is lower when the destination node is farther away in the network. We will explore this last hypothesis to examine whether there are differences in memory structures between children and adults.

A network of nodes is assumed to be acquired by learning. Learning consists of acquiring new knowledge, incorporating new facts into prior knowledge, or modifying organizational structures (Rumelhart \& Norman, 1981). A crucial question is: How can these organizational properties of knowledge be captured and represented?

Relation and similarity judgments have been used in the area of cognitive psychology to assess the content of semantic memory. This procedure typically produces a matrix of proximity values in which each value represents the degree of relationship of a pair of concepts. The Schvaneveldt research group (Schvaneveldt, 1990; Schvaneveldt, Dearholt, \& Durso, 1988; Schvaneveldt, Durso, \& Dearholt, 1989) has developed a graph-theoretical technique (Pathfinder) that derives network structures from proximity data.

The Pathfinder algorithm transforms a proximity matrix into a network structure in which each object is represented by a node in the network and the relationship between objects is depicted by how closely they are linked. The method searches through the network nodes for the closest indirect path between objects. A direct link between two nodes is added only if the closest indirect path between them is greater than the proximity value for that pair of objects. One of Pathfinder's strengths is that it does not force hierarchical solutions, although hierarchical networks may emerge if the data is organized hierarchically.

Three measures can be used with Pathfinder structures: (a) number of links in the network, (b) a closeness value $(C)$, and (c) graph-theoretical distances (GTDs, number of links between pairs of concepts). The $C$ measure $^{2}$ is a settheoretical method of quantifying the similarity between two networks having a common set of nodes. Briefly, $C$ examines the degree to which the same node in two graphs is surrounded by a similar neighborhood of nodes. This neighborhood comparison is performed for each node in the two graphs and the results are averaged across the nodes to compute an overall similarity index. $C$ values range from 0 , for complementary graphs, to 1 , for identical graphs (Goldsmith, Johnson, \& Acton, 1991).

Possible differences in children's and adults' structures can be studied with this method, which captures the relation structure of a set of concepts. Children's concept structures may have fewer links than those of adults. If so, in adults' networks, more activation would accumulate for a node because it would receive more activation from its links. The distance between prime node and target node may be large in children's networks and small in adults' networks. If so, as distance between the prime and target node increases, activation received by the target node would be lower.

\section{Experiment 1}

In this experiment, memory structure representations were measured by Pathfinder algorithm. The relatedness of judgments for homographs and their associates (words that a subject sample has emitted in a free association task-see Table 1) was recorded to obtain Pathfinder network structure representations (see Figure 1 for an example). Various groups of participants were selected to detect developmental changes in these representations.

As mentioned above, with this procedure, age-related changes in representational memory structures can be examined. Some variables, such as printed usage frequency, structure type, and associative strength were introduced in the analysis to detect their relations with Pathfinder networks.

\section{Method}

Participants. A total of 35 individuals participated in this study. There were 5 volunteers in the Professor Group, selected from lecturers at the University of Almería, all of the same age $(M=32, S D=0)$. Although five may seem a small number for a group, previous studies also using a small number of experts provided stable referent structures with which to compare students (e.g., Goldsmith et al., 1991).

Ten volunteers from a teacher training group, with a mean age of 19.6 years $(S D=2.88)$ were selected from first-year students at the University of Almería, as the University Group. Another 10 volunteers, with a mean age of 13.3 years $(S D=0.48)$, from the second-year high school students at the Caravaca High School (Almería),

\footnotetext{
2 Computer programs for performing Pathfinder and $\mathrm{C}$ are available for several different computers. Information about obtaining programs is available from Interlink, Inc., P. O. Box 4086 UPB, Las Cruces, New Mexico 88003-4086.
} 
made up the Secondary Group. Lastly, 10 volunteers of the same age $(M=11, S D=0)$ were chosen from the sixth grade at the Goya School (Almería), for the Primary Group.

Except for the Professor Group, in each group, the participants were randomly selected from a larger pool. All participants' measures were recorded during the first months of 1999.

Stimuli. Ten homographs were selected from Nievas and Cañas's (1993) association norms. Nine of the homographs were the same as Nievas (1999a, 1999b; see Appendix B) used in his semantic priming experiment with children and adults. For each homograph, all the associates that had associative strength higher than $1 \%$ in the association norms were chosen. Only five associates with $2 \%$ of associative strength were not selected due to control. Therefore, 114 concepts were selected for ratings, including the homographs. Each homograph had an average of 11.4 associated concepts $(S D=1.5)$.

Five homographs had a low printed usage frequency (PUF), $M=1.16, S D=2.59$, measured in units per million (Juilland \& Chang-Rodríguez, 1964). Any homograph not present in this frequency study was assigned a zero value.
The other five homographs had a medium PUF $(M=39.54$, $S D=41.3$ ). Each homograph, both in low and medium PUF, had a mean of 11.4 nodes ( $S D=1.5$ and 1.7 , respectively).

Procedure. Participants were requested to rate the semantic relatedness of pairs of concepts for ten blocks (each block was formed by the homograph and its associates). Each participant performed 603 ratings. Each block had an average of 60.3 ratings $(S D=16.5)$.

Participants rated each pair of concepts on a 9-point Likert-type response formatted scale ranging from least related (1) to most related (9). Each pair of concepts was centered from left to right on an 80486-DX personal computer screen. The order in which the pairs for each block were presented was randomized for each participant. Participants responded by pressing the appropriately numbered key on the number keyboard. On average, participants needed approximately one hour to complete the rating task. Each participant was tested individually. At the beginning of the task, participants were instructed that they could ask the meaning of any word they did not know. Only two participants in the primary group asked the meaning of espinilla [blackhead; shin].

Table 1

Associative Strength (Percentage of Participants that Respond the Same Word) for Associates from a Homograph

\begin{tabular}{|c|c|c|c|}
\hline Dominant meaning & $\%$ & Subordinate meaning & $\%$ \\
\hline adelgazar (to slim) & 15.7 & dictadura (dictatorship) & 9.8 \\
\hline dieta (diet) & 9.8 & política (political) & 8.8 \\
\hline comida (meal) & 7.8 & dictador (dictator) & 2.0 \\
\hline hambre (hunger) & 6.9 & abierto (open) & 2.0 \\
\hline gordo (fat) & 5.9 & democrático (democratic) & 2.0 \\
\hline gordura (fatness) & 1.0 & Franco (Spanish dictator) & 2.0 \\
\hline lechuga (lettuce) & 1.0 & sociedad (society) & 1.0 \\
\hline disciplina (discipline) & 1.0 & militar (military) & 1.0 \\
\hline plan (plan) & 1.0 & liberal (liberal) & 1.0 \\
\hline nutrición (nutrition) & 1.0 & fascista (fascist) & 1.0 \\
\hline obligación (obligation) & 1.0 & condena (condemnation) & 1.0 \\
\hline alimentar (to feed) & 1.0 & antiguo (former) & 1.0 \\
\hline delgado (slim) & 1.0 & interno (internal) & 1.0 \\
\hline adelgazamiento (slimming) & 1.0 & forma (form) & 1.0 \\
\hline alimenticio (nutritious) & 1.0 & agrario (agrarian) & 1.0 \\
\hline extinción (extinction) & 1.0 & & \\
\hline comer (to eat) & 1.0 & & \\
\hline verduras (vegetables) & 1.0 & & \\
\hline engordar (to get fat) & 1.0 & & \\
\hline anorexia (anorexia) & 1.0 & & \\
\hline fuerza (strength) & 1.0 & & \\
\hline duro (hard) & 1.0 & & \\
\hline Total & 62.80 & Total & 35.30 \\
\hline Blank & 2.0 & & \\
\hline
\end{tabular}

Note. In free associative tasks, a (homograph) word was presented and the participant had to write down the first word that came to mind. This example was taken from the Nievas and Cañas (1993) norms. 


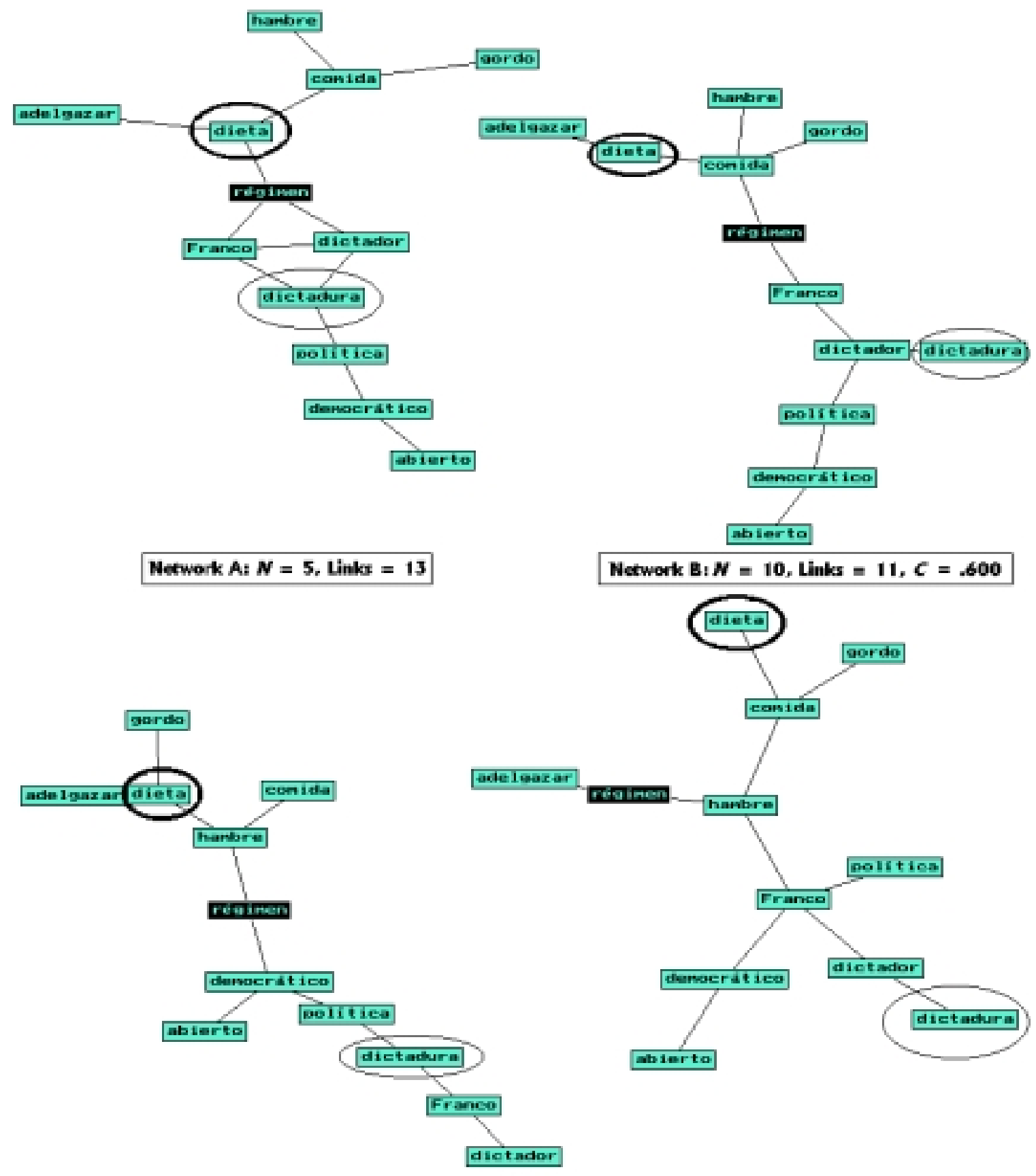

Network $C_{t} N=10$, Links $=11, C=.412$

Network $D: N=10$, Links $=11, C=.353$

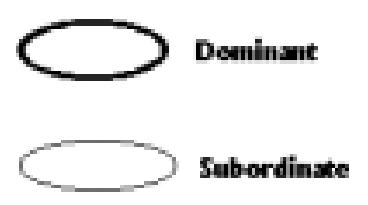

\section{Network At Professor Group \\ Network B: University Greup \\ Netwerk C: Secendary Group \\ Network D: Primary Group}

Figure 1. An illustration of the memory structure network representations for the homograph régimen. Note that each network was computed by the average rating matrix, so that they are average network representations. 
Design. Pathfinder analysis reduces large amounts of proximity data to interpretable forms, thus generating a network representation of the concepts. The strength of the association between two concepts is represented by their proximity, that is, the longer the distance, the lower the proximity, and vice versa. Two concepts are directly connected in the network if, and only if, the distance of their direct link is shorter than all of their indirect links in the network.

A variety of Pathfinder networks can be derived from a given set of proximity data. A particular network is determined by the values of two parameters, $r$ and $q$, which represent generalizations of the usual definition of distances in networks. The parameter $r$ determines the way the weight of a path is computed from the weights on links in the path, whereas $q$ limits the number of links allowed in paths. For all participants and for each homograph block with its associates, we used $r=$ infinite and $q=n-1$ for one condition. These parameter values produce the smallest number of links in Pathfinder networks (Schvaneveldt et al., 1989). The other condition, $r=$ infinite and $q=2$, produces a greater number of links in Pathfinder networks.

A mixed factorial ANOVA was used for each of the three dependent variables: (a) number of links, (b) $C$ measures, and (c) distances between primes and targets. Printed Usage Frequency (PUF) (low and medium), and $q$ parameter ( $q=$ $n-1$ and $q=2$ ) were manipulated within subjects. The Age Group (Primary, Secondary, University, and Professors) was manipulated between subjects. A new factor was introduced for distances: Dominance (Dominant and Subordinate), manipulated within subjects, because targets can be either dominant (belonging to the more frequent meaning) or subordinate (belonging to a less frequent meaning).

\section{Results}

As mentioned, the concept ratings were analyzed by a Pathfinder algorithm with $r=$ infinite and $q=n-1$ for one condition, and $r=$ infinite and $q=2$ for the other, for each participant and each homograph block. Therefore, for each participant, twenty networks were obtained, one for each homograph with its associates. Ten were for $r=$ infinite and $q=n-1$ and the other ten were for $r=$ infinite and $q=2$.

Number of links. The main effect for Age Group was nonsignificant, $F(3,31)=1.857, M S_{\mathrm{e}}=117.734, p=.158$, which shows that there were no differences in the number of links as age increased. The effect for PUF was also nonsignificant, $F(1,31)=2.165, M S_{\mathrm{e}}=10.74, p=.151$, that is, the Low PUF Group did not have fewer links than the Medium PUF Group.The PUF $\times$ Age Group interaction was nonsignificant, $\mathrm{F} \approx 1$. The main effect for $q$ parameter was significant, $F(1,31)=120.8, M S_{\mathrm{e}}=7.53, p=.000$. Therefore, for $q=2$, there were more links than for $q=n$ - 1. The $q$ parameter $\times$ Age Group interaction was marginally significant, $F(3,31)=2.427, M S_{\mathrm{e}}=7.53, p=$ .084. All the remaining effects had $F \approx 1$.

We carried out a separate ANOVA for each condition of $q$ parameter because $q$ parameter $\times$ Age Group interaction was marginally significant. For the $q=n-1$ condition, the main effect for Age Group was nonsignificant, $F(3,31)=$ $1.235, M S_{\mathrm{e}}=57.783, p=.314$. The main effect for PUF was marginally significant, $F(1,31)=3.452, M S_{\mathrm{e}}=5.4, p$ $=.073$. The Medium PUF Group had more links than the Low PUF Group. All the remaining effects had $F \approx 1$. For the $q=2$ condition, the main effect for Age Group was marginally significant, $F(3,31)=2.45, M S_{\mathrm{e}}=67.48, p=$ .082 , that is, as age increased, there were fewer links. All the remaining effects were nonsignificant $(F \approx 1)$.

When $q=n-1$, there were no differences between groups, but when $q=2$ there were more links as age decreased. As seen in Table 2, the Professor Group seems to be responsible for the significant values. Therefore, we carried out the comparison of Age Group (Primary, Secondary, and University) without the Professor Group. For $q=2, F(2,27)<1$, that is, there were no differences in the number of links between these groups. Another comparison revealed differences between the Professor Group and the University Group for $q=2, F(1,13)=5.447, M S_{\mathrm{e}}$ $=66.3, p=.036$, with the Professor Group having fewer links than the University Group.

Table 2

Means and Standard Errors (in Brackets) of the Dependent Variable Number of Links as a Function of Age Group, $q$ Parameter, and Printed Usage Frequency (PUF) for Experiment 1

$q$ Parameter

\begin{tabular}{lcccr}
\multirow{2}{*}{ Age Group } & \multicolumn{2}{c}{$q=2$} \\
\cline { 2 - 5 } & Low PUF & Medium PUF & Low PUF & Medium PUF \\
\hline Primary Group & $20.1(1.9)$ & $21.2(1.6)$ & $27.5(2.0)$ & $27.6(1.8)$ \\
Secondary Group & $21.4(1.9)$ & $24.0(1.6)$ & $26.6(2.0)$ & $29.4(1.8)$ \\
University Group & $21.2(1.9)$ & $21.3(1.6)$ & $27.7(2.0)$ & $27.1(1.8)$ \\
Professor Group & $16.8(2.7)$ & $17.4(2.3)$ & $20.0(2.8)$ & $20.2(2.6)$ \\
\hline
\end{tabular}


With $q=n-1$, we compared Age Group (Primary, Secondary, and University) in PUF effect, revealing that Low PUF had fewer links than did Medium PUF, $F(1,27)$ $=3.81, M S_{\mathrm{e}}=6.05, p=.061$.

$C$ measures. $C$ values were obtained for each participant and each homograph. As mentioned, the $C$ value provides a measure of the degree of similarity between two graphs, that is, a measure of the extent to which the nodes in two graphs are surrounded by a similar set of nodes. We computed an average matrix of ratings for each homograph from participants in the Professor Group. With these average data matrixes, the average Pathfinder network was calculated for each homograph. These networks were used to find the $C$ values for each participant and each homograph in each group.

The effect for Age Group was statistically significant, $F(3,31)=28.076, M S_{\mathrm{e}}=0.007, p=.000$, and, as age increased, we found participant networks more similar to the average professor network for each homograph. PUF was also significant, $F(1,31)=6.4, M S_{\mathrm{e}}=0.001, p=.017$. Therefore, the low PUF networks were more similar to the average professor networks than were the medium PUF networks. There was a main effect for $q$ parameter, $F(1,31)$ $=9.679, M S_{\mathrm{e}}=0.001, p=.004$, that is, $C$ values were higher when $q=n-1$ than when $q=2$. All the remaining effects had $F \approx 1$. As seen in Table 3, the Professor Group again seems to be responsible for the differences. We therefore carried out an ANOVA without the Professor Group. The main effect for Age Group was marginally significant, $F(2$, $27)=2.823, M S_{\mathrm{e}}=0.006, p=.077$. The main effect for $q$ parameter was significant, $F(1,27)=12.482, M S_{\mathrm{e}}=0.001$, $p=.002$. The main effect for PUF was also significant, $F(1$, $27)=13.310, M S_{\mathrm{e}}=0.001, p<.001$. These results showed that the Professor Group did not affect the variables.

As interactions $q$ parameter $\times$ Age Group and PUF $\times$ Age Group were both marginally significant, $F(2,27)=1.9, M S_{\mathrm{e}}$ $=0.001, p=.167$, and $F(2,27)=2.1, M S_{\mathrm{e}}=0.001, p=.142$, respectively, we carried out separate ANOVAs for $q=n-1$ and $q=2$. For $q=n-1$, and four groups in Age Group, the main effect for Age Group was statistically significant, $F(3$, $31)=26.045, M S_{\mathrm{e}}=0.004, p=.000$. The main effect for PUF was marginally significant, $F(1,31)=3.180, M S_{\mathrm{e}}=0.001, p$ $=.084$. Removing the Professor Group from the Age Group, the main effect for Age Group was significant, $F(2,27)=4.631$, $M S_{\mathrm{e}}=0.003, p=.019$, as was the main effect for PUF, $F(1$, $27)=8.091, M S_{\mathrm{e}}=0.001, p=.008$.

With $q=2$, and all four groups in Age Group, there was a statistically significant main effect for Age Group, $F(3,31)$ $=25.527, M S_{\mathrm{e}}=0.004, p=.000$, and for PUF, $F(1,31)=$ 7.893, $M S_{\mathrm{e}}=0.001, p=.009$. Removing the Professor Group from the Age Group, the main effect for Age Group was nonsignificant, $F \approx 1$. The main effect for PUF was significant, $F(1,27)=13.206, M S_{\mathrm{e}}=0.000, p<.001$.

Table 3

Means and Standard Errors (in Brackets) of the Dependent Variable C Value as a Function of Age Group, $q$ Parameter, and Printed Usage Frequency (PUF) for Experiment 1

\begin{tabular}{lrrrr}
\hline & & & $q$ Parameter & \\
Age Group & \multicolumn{2}{c}{$q=n-1$} & & $q=2$ \\
\cline { 2 - 5 } & Low PUF & Medium PUF & Low PUF & Medium PUF \\
\hline Primary Group & $.327(.014)$ & $.308(.017)$ & $.322(.014)$ & $.301(.017)$ \\
Secondary Group & $.367(.014)$ & $.330(.017)$ & $.345(.014)$ & $.315(.017)$ \\
University Group & $.374(.014)$ & $.368(.017)$ & $.344(.014)$ & $.337(.017)$ \\
Professor Group & $.521(.019)$ & $.528(.024)$ & $.526(.020)$ & $.514(.024)$ \\
\hline
\end{tabular}

Table 4

Means and Standard Errors (in Brackets) of the Dependent Variable Distances as a Function of Age Group, q Parameter, Printed Usage Frequency (PUF), and Dominance for Experiment 1

\begin{tabular}{|c|c|c|c|c|c|c|c|c|}
\hline \multirow{4}{*}{ Age Group } & \multicolumn{8}{|c|}{$q$ Parameter } \\
\hline & \multicolumn{4}{|c|}{$q=n-1$} & \multicolumn{4}{|c|}{$q=2$} \\
\hline & \multicolumn{2}{|c|}{ Low PUF } & \multicolumn{2}{|c|}{ Medium PUF } & \multicolumn{2}{|c|}{ Low PUF } & \multicolumn{2}{|c|}{ Medium PUF } \\
\hline & Dominant & Subordinate & Dominant & Subordinate & Dominant & Subordinate & Dominant & Subordinate \\
\hline Primary Group & $1.82(0.12)$ & $2.00(0.12)$ & $1.84(0.10)$ & $1.68(0.10)$ & $1.44(0.07)$ & $1.52(0.08)$ & $1.42(0.08)$ & $1.50(0.07)$ \\
\hline Secondary Group & $1.92(0.12)$ & $1.50(0.12)$ & $1.68(0.10)$ & $1.44(0.10)$ & $1.58(0.07)$ & $1.46(0.08)$ & $1.34(0.08)$ & $1.34(0.07)$ \\
\hline University Group & $1.64(0.12)$ & $1.74(0.12)$ & $1.58(0.10)$ & $1.52(0.10)$ & $1.42(0.07)$ & $1.50(0.08)$ & $1.34(0.08)$ & $1.30(0.07)$ \\
\hline Professor Group & $1.60(0.17)$ & $1.60(0.16)$ & $1.32(0.14)$ & $1.40(0.14)$ & $1.52(0.11)$ & $1.52(0.11)$ & $1.20(0.11)$ & $1.32(0.10)$ \\
\hline
\end{tabular}


Distances. Distances were computed from homograph networks for each participant. Distances were calculated by the number of intermediate links between the homograph and two associates. One associate belongs to one meaning of the homograph word and the other to another meaning of the homograph. These two associates were the dominant and subordinate.

The main effect for Age Group was marginally significant, $F(3,31)=2.537, M S_{\mathrm{e}}=0.204, p=.075$, revealing that, as age increased, the distances between homographs and targets were shorter. PUF was also statistically significant, $F(1,31)=20.996, M S_{\mathrm{e}}=0.078, p$ $=.000$, that is, as frequency increased, distances were shorter. The main effect for dominance was nonsignificant, $F<1$. The main effect for $q$ parameter was significant, $F(1,31)=68.1, M S_{\mathrm{e}}=0.046, p=.000$ : With $q=n-1$, the distances were greater than with $q=2$. The $q$ parameter $\times$ Age Group interaction was significant, $F(3,31)=4.04$, $M S_{\mathrm{e}}=0.046, p=.015$. We therefore carried out separate ANOVAs for $q=n-1$ and $q=2$. For $q=n-1$, significant main effects were revealed for Age Group, $F(3,31)=3.789$, $M S_{\mathrm{e}}=0.172, p=.020$, and PUF, $F(1,31)=17.4, M S_{\mathrm{e}}$ $=0.053, p=.000$, respectively. All the remaining effects had $\mathrm{F} \approx 1$.

For $q=2$, whereas the main effect for Age Group was nonsignificant, $F<1$, the main effect for PUF was significant, $F(1,31)=15.20, M S_{\mathrm{e}}=0.047, p=.000$. Considering the Professor Group as not being a genuine group, separate ANOVAs for $q=n-1$ and $q=2$ and only three groups (Primary, Secondary, and University) were performed. For $q=n-1$, the main effect for Age Group was marginally significant, $F(2,27)=3.084, M S_{\mathrm{e}}=0.187$, $p=.062$, and the main effect for PUF was significant, $F(1$, $27)=10.93, M S_{\mathrm{e}}=0.059, p=.003$. For $q=2$, only PUF was significant $F(1,27)=8.028, M S_{\mathrm{e}}=0.048, p=.009$. Hence, using three groups (Primary, Secondary, and University) obtained the same results as using four groups (Primary, Secondary, University, and Professor).

\section{Discussion}

The results of the first experiment suggest that there are some age-related differences in the organization of semantic knowledge, as measured by Pathfinder representations. That is, the effect of Age Group on $C$ measures was significant for less complex structures ( $q=n-1$, fewer links), both for Age Group with three groups (Primary, Secondary, and University) and for Age Group with four groups (Primary, Secondary, University, and Professor). For more complex structures ( $q=2$, more links), Age Group was significant if the Professor Group was included. If the Professor Group was excluded, then there were no differences in $C$ measures. As mentioned above, the Pathfinder algorithm is assumed to capture relations among concept words, that is, the organizational properties of knowledge. Some studies show that the Pathfinder algorithm is better than the multidimensional scaling technique for capturing these fine relations (Goldsmith et al., 1991; Gonzalvo, Cañas, \& Bajo, 1994). Thus, there seems to be a developmental change in the organization of semantic knowledge representations. Conceptual structure representations of homographs were significantly different as age increased: At higher ages, these representations between homographs and their associates were more similar to the professors' average conceptual structure representations. Thus, semantic memory structure representations may be dynamic, with concept organizational structures that are continuously updated as age increases, for less complex structures ( $q=n-1$, fewer links).

This result — changes with age in concept organizational structures-supports Rumelhart and Norman's (1981) point of view of learning, because modification of the organizational structures is a source of learning. This result also supports some new approaches to conceptual change (Schnotz, Vosniadou, \& Carretero, 1999).

Distances provide another source of age-related differences. With less complex structures $(q=n-1$, fewer links), there was a significant Age Group effect for the four groups (Primary, Secondary, University, and Professor). That is, as age increased the distances were shorter. But with more complex structures $(q=2$, more links), the effect for Age Group was nonsignificant. That is, with complex structures-more links-, there are more ways to reach the target by one path or another, but with less complex structures-fewer links-, there are fewer paths to reach the target word. Spreading activation theories predict that, as distances between prime and target nodes in an individual's network increase, the amount of activation received in the target node from the prime node will decrease, because activation decays over time and space. Thus, a response to the issue of why there is no semantic priming in younger people might be because prime and target distances may be greater in younger people's conceptual networks, whereas for adults they could be relatively short. To check this hypothesis, it was assumed that the Pathfinder algorithm can capture these distances. Age Group had a significant effect on the distances between prime and target when $q=n-1$ (fewer links). That is, as age increased, the distances between prime and target were smaller.

Another finding was that there are no differences between the groups in the number of links between networks measured by the Pathfinder method except for the Professor Group, with more complex structures $(q=$ 2 ), which had fewer links than the other groups. We assumed that missing semantic priming with homographs in younger people could be due to there being fewer links between concepts in children. These reduced links, as compared with those of adults, could have provided less activation to a target word, and therefore, no semantic priming. We assumed that semantic priming is a 
consequence of the spreading activation of the prime. So, when a target word is presented, less activation is needed to recognize it because the target node has already received activation from the prime node. But if there were more links between the prime and target, priming would probably be higher because there would be more paths to transmit activation. However, the results are clear. There are no differences between younger people and adults in the number of links in the concept structure using Pathfinder representations, except for the Professor Group.

Another relevant finding is related to the effect of PUF on the number of links as dependent variable. The PUF effect appears with less complex structures $(q=n-1)$, and the Low PUF Group had fewer links than the Medium PUF Group. In other words, the number of links in the homograph network, measured by Pathfinder, is related to PUF. As a word is processed, it is more likely to enable more links with other words. But the PUF effect was nonsignificant with more complex structures ( $q=2$, more links).

Moreover, low frequency networks were more similar to the average professor networks than were medium frequency networks, measured by $C$ values. That is, as frequency increases, organizational structures are more likely to be complex and, thus, different. This statement is supported by the finding that high frequency words have many meanings compared with low frequency words (Glanzer \& Bowles, 1976; Reder, Anderson, \& Bjork, 1974; Schorr \& Atkinson, 1970). Similarly, distances between prime (homograph) word and target words were higher in the Low PUF Group than in the Medium PUF Group. This result suggests that the PUF of the prime word plays a role in the degree of relatedness between prime and target words. As frequency increases, there may be more opportunities to relate prime and target words, and therefore, for their becoming more strongly related.

Lastly, a result connects associative strength, measured by associative norms, with distances, measured by the Pathfinder algorithm. Distances between prime words (homographs) and target words were not higher for dominant targets than for subordinate targets. It should be noted that the associative strength was $8.3 \%$ for dominant target words, and $8.2 \%$ for subordinate target words, practically the same.

\section{Experiment 2}

This second experiment was designed to test whether Pathfinder measures and earlier effects were consistent across different participant groups, and whether those changes across ages in organizational memory structure representations were due to cohort effects. For example, changes in Pathfinder networks between the secondary and the university group might emerge because each group was exposed to different kinds of stimuli. In other words, will two groups of the same age have the same Pathfinder networks, testing one group in 1994 and the other in 1999 ?

\section{Method}

Participants. Ten volunteer students, mean age 19.2 years $(S D=1.92)$, were selected from the first-year Introductory Psychology course at the University of Granada, to make up the Granada University Group. The participants were chosen randomly from a larger pool. In this group, all participants' measures were recorded during the first months of 1994. The second group was the Almería University Group (University Group) from Experiment 1. Stimuli and procedure were the same as in Experiment 1.

Design. A mixed factorial ANOVA was used for each of the three dependent variables: (a) number of links, (b) $C$ measures, and (c) distances between prime and targets. Printed Usage Frequency (PUF) (low and medium) and $q$ parameter $(q=n-1$ and $q=2)$ were manipulated within subjects. Age Group (Granada University Group and Almería University Group) was manipulated between subjects. Dominance (Dominant and Subordinate) was manipulated within subjects.

\section{Results}

Concept ratings were analyzed by a Pathfinder algorithm with $r=$ infinite and $q=n-1$ and $r=$ infinite and $q=2$ for each participant and each homograph block. Therefore, for each participant, 20 networks were obtained, one for each homograph with its associates.

Table 5

Means and Standard Errors (in Brackets) of the Dependent Variable Number of Links as a Function of Age Group, $q$ Parameter, and Printed Usage Frequency (PUF) for Experiment 2

\begin{tabular}{|c|c|c|c|c|}
\hline \multirow{3}{*}{ Group } & \multicolumn{4}{|c|}{$q$ Parameter } \\
\hline & \multicolumn{2}{|c|}{$q=n-1$} & \multicolumn{2}{|c|}{$q=2$} \\
\hline & Low PUF & Medium PUF & Low PUF & Medium PUF \\
\hline Almería University Group & $21.22(1.94)$ & $21.34(1.40)$ & $27.74(2.00)$ & $27.14(1.57)$ \\
\hline Granada University Group & $21.14(1.94)$ & $21.28(1.40)$ & $25.56(2.00)$ & $25.48(1.57)$ \\
\hline
\end{tabular}


Number of links. The main effect for Age Group was statistically nonsignificant $(\mathrm{F}<1)$, which indicates that there were no differences in the number of links between the Granada and Almería University groups. The $q$ parameter effect was statistically significant, $F(1,18)=$ $85.188, M S_{\mathrm{e}}=6.434, p=.000$, that is, with $q=2$, there were more links than with $q=n-1$. The $q$ parameter $\times$ Age Group interaction was marginally significant, $F(1,18)$ $=2.66, M S_{\mathrm{e}}=6.434, p=.120$. Separate ANOVAs were carried out for $q=n-1$ and $q=2$, revealing no significant effects $(\mathrm{F} \approx 1)$.

$C$ measures. $C$ values were obtained for each participant and each homograph for $q=\mathrm{n}-1$ and $q=2$. We computed an average matrix of ratings for each homograph from the Professor Group in Experiment 1. With these average data matrixes, the average Pathfinder network was calculated for each homograph. These networks were used to find $C$ values for each participant and each homograph in each group.

The effect for Age Group was marginally significant, $F(1,18)=3.872, M S_{\mathrm{e}}=0.002, p=.065$, that is, $C$ measures were higher for the Granada University group. The main effect for $q$ parameter was also significant, $F(1,18)=12.337$, $M S_{\mathrm{e}}=0.001, p=.002$, revealing that, with $q=2, C$ measures were lower than in the $q=n-1$ condition. The $q$ parameter $\times$ Age Group interaction was marginally significant, $F(1,18)=2.176, M S_{\mathrm{e}}=0.001, p=.157$. The main effect for PUF was significant, $F(1,18)=4.94, M S_{\mathrm{e}}$
$=0.002, p=.039$. Therefore, low frequency networks were more similar to the average professor networks than were the medium frequency networks. The interaction PUF $\times$ Age Group was marginally significant, $F(1,18)=2.529$, $M S_{\mathrm{e}}=0.002, p=.129$. We carried out separate ANOVAs for $q=n-1$ and $q=2$. For $q=n-1$, the Age Group main effect was nonsignificant $(\mathrm{F}<1)$ and the PUF effect was marginally significant, $F(1,18)=3.466, M S_{\mathrm{e}}=0.001, p=$ .079 . For $q=2$, there was a significant main effect for Age Group, $F(1,18)=5.3, M S_{\mathrm{e}}=0.002, p=.033$, and for PUF, $F(1,18)=4.824, M S_{\mathrm{e}}=0.001, p=.042$

Distances. The main effect for the $q$ parameter was statistically significant, $F(1,18)=55.993, M S_{\mathrm{e}}=0.026, p$ $=.000$, revealing that less complex structures $(q=n-1$, fewer links) had greater distances than did more complex structures $(q=2$, more links). The $q$ parameter $\times$ Age Group interaction was marginally significant, $F(1,18)=2.125$, $M S_{\mathrm{e}}=0.026, p=.162$. The main effect for PUF was significant, $F(1,18)=4.401, M S_{\mathrm{e}}=0.105, p=.050$, revealing shorter distances as frequency increased. We carried out ANOVAs for $q$ parameter. For $q=n-1$, the main effect for Age Group was nonsignificant, $F(1,18)=1.976, M S_{\mathrm{e}}$ $=0.146, p=.177$, whereas the main effect for PUF was marginally significant, $F(1,18)=4.285, M S_{\mathrm{e}}=0.073, p=$ .053 . For $q=2$, the main effect for Age Group was again nonsignificant $(\mathrm{F}<1)$ and the main effect for PUF was marginally significant $F(1,18)=3.078, M S_{\mathrm{e}}=0.047, p=$ .096. All the remaining effects had $\mathrm{F} \approx 1$.

Table 6

Means and Standard Errors (in Brackets) of the Dependent Variable C Values as a Function of Age Group, q Parameter, and Printed Usage Frequency (PUF) for Experiment 2

\begin{tabular}{lrrrr}
\hline \multirow{2}{*}{ Group } & \multicolumn{2}{c}{$q$ Parameter } & & $q=2$ \\
\cline { 2 - 5 } & Low PUF & Medium PUF & Low PUF & Medium PUF \\
\hline Almería University Group & $.374(.014)$ & $.368(.009)$ & $.344(.012)$ & $.337(.012)$ \\
Granada University Group & $.401(.014)$ & $.363(.009)$ & $.389(.012)$ & $.350(.012)$ \\
\hline
\end{tabular}

Table 7

Means and Standard Errors (in Brackets) of the Dependent Variable Distances as a Function of Age Group, q Parameter, Printed Usage Frequency (PUF), and Dominance for Experiment 2

\begin{tabular}{|c|c|c|c|c|c|c|c|c|}
\hline \multirow{4}{*}{ Group } & \multicolumn{8}{|c|}{$q$ Parameter } \\
\hline & \multicolumn{4}{|c|}{$q=n-1$} & \multicolumn{4}{|c|}{$q=2$} \\
\hline & \multicolumn{2}{|c|}{ Low PUF } & \multicolumn{2}{|c|}{ Medium PUF } & \multicolumn{2}{|c|}{ Low PUF } & \multicolumn{2}{|c|}{ Medium PUF } \\
\hline & Dominant & Subordinate & Dominant & Subordinate & Dominant & Subordinate & Dominant & Subordinate \\
\hline \multicolumn{9}{|l|}{ Almería } \\
\hline $\begin{array}{l}\text { University Group } \\
\text { Granada }\end{array}$ & $1.64(0.10)$ & $1.74(0.12)$ & $1.58(0.09)$ & $1.52(0.12)$ & $1.42(0.07)$ & $1.50(0.07)$ & $1.34(0.06)$ & $1.30(0.09)$ \\
\hline University Group & $1.52(0.10)$ & $1.60(0.12)$ & $1.48(0.09)$ & $1.40(0.12)$ & $1.36(0.07)$ & $1.36(0.07)$ & $1.32(0.06)$ & $1.34(0.09)$ \\
\hline
\end{tabular}




\section{Discussion}

These data showed that there was no cohort effect, that is, the differences between Almería University Group and Granada University Group were nonsignificant. This was true for the dependent variable number of links and for distances: There were no differences between the groups. However, in $C$ measures there were differences. With $q=$ $n-1$ (fewer links), there were no differences between the groups, but with $q=2$ (more links), the Granada University Group had higher $C$ measures. Lastly, $q$ parameter effect was present in the three dependent variables. In number of links, more complex structures had more links than less complex ones. In $C$ measures, more complex structures had lower $C$ s. In distance, more complex structures had lower distances. Therefore, this second experiment showed that, in number of links and distances, there were no differences between the two groups, one measured in 1994 and the other in 1991. In $C$ measures, there were only differences with $q=2$.

The difference between the Granada and Almería University Groups in $C$ values for $q=2$ could be due to the fact that, with $q=2$, the structures have more links than with $q=n-1$; therefore, the more complex structures were more likely to be different.

There were PUF effects on the two dependent variables, that is, $C$ values and distances, but not on number of links.

\section{Experiment 3}

Results from Experiment 1 showed that Pathfinder distances were different in younger and adult people. Distances between primes and targets were smaller as age increased. Are Pathfinder distances related to any variable that is used in semantic priming experiments? The aim of Experiment 3 was to examine the relation between Pathfinder distances and associative strength, measured by the associative norms that are usual in semantic priming experiments.

\section{Method}

Participants. A total of 60 individuals participated in this experiment. They were volunteers from an Introductory Psychology course at the University of Granada. These students were measured during their first year at the university, so that they were approximately 19 years old.

Stimuli. Fifty-six homographs were selected from the association norms of Nievas and Cañas (1993). All the homograph associates with associative strength higher than $1 \%$ in the association norms were chosen for each homograph. Therefore, 746 concepts were selected for ratings, including the homographs.
Design. Six blocks were formed with the homographs: two blocks with ten homographs and four blocks with nine homographs. Each block was rated by 10 participants. Therefore, each homograph with its associates was rated by 10 participants. We always used $r=$ infinite and $q=n-1$. Distances between each homograph and its associates were taken from the average rating matrix of the 10 participants.

An ANOVA was carried out. The dependent variable was the associative strength between each homograph and each of its associates that was extracted from Nievas and Cañas' (1993) associative norms. Distances were calculated for each homograph and its associates, and these were manipulated. They ranged from 1 (no intermediate nodes between the homograph and its associate) to 9 (eight intermediate nodes).

Another ANOVA used as dependent variable the associative strength between each homograph and each of its associates. Truncated average ratings were the average of the degree of relationship between each homograph and each of its associates from 10 participants, without decimals. These ratings were manipulated and they ranged from 1 (least related) to 9 (most related).

Procedure. The procedure was the same as in the previous experiments.

Results

Pathfinder distance. The concept ratings were analyzed by a Pathfinder algorithm with $r=$ infinite and $q=n-1$ for the average rating matrixes of each homograph. Therefore, each homograph with its associates had one Pathfinder network that was an average from 10 participants. As seen in Table 8, the main effect for distance was statistically significant, $F(8,681)=5.536, M S_{\mathrm{e}}=57.416, p$ $=.000$, revealing that, as distances increased, the associative strength was lower.

Table 8

Means and Standard Errors (in Brackets) of the Associative Strength in Percentages as a Function of the Distance Between the Homograph and its Associate, and Number of Associates with the Same Distance (N) for Experiment 3

\begin{tabular}{ccr}
\hline Distance & $M \quad(S D)$ & $N$ \\
\hline 1 & $9.239(0.570)$ & 177 \\
2 & $5.784(0.527)$ & 207 \\
3 & $4.927(0.613)$ & 153 \\
4 & $4.263(0.832)$ & 83 \\
5 & $4.371(1.246)$ & 37 \\
6 & $3.967(1.654)$ & 21 \\
7 & $3.360(2.864)$ & 7 \\
8 & $5.227(4.375)$ & 3 \\
9 & $2.450(5.358)$ & 2 \\
\hline
\end{tabular}


Table 9

Means and Standard Errors (in Brackets) of the Associative Strength in Percentages as a Function of the Truncated Average Rating Between the Homograph and its Associate and Number of Associates with the Same Rating (N) for Experiment 3

\begin{tabular}{ccr}
\hline Truncated average rating & $M(S D)$ & $N$ \\
\hline 1 & $1.960(3.603)$ & 4 \\
2 & $4.352(1.441)$ & 25 \\
3 & $3.966(1.169)$ & 38 \\
4 & $3.806(0.777)$ & 86 \\
5 & $4.478(0.714)$ & 102 \\
6 & $5.381(0.577)$ & 156 \\
7 & $6.434(0.586)$ & 151 \\
8 & $9.634(0.655)$ & 121 \\
9 & $28.851(2.724)$ & 7 \\
\hline
\end{tabular}

Relation judgments. The main effect for the truncated average ratings was statistically significant, $F(8,681)=$ $15.098, M S_{\mathrm{e}}=51.938, p=.000$, revealing that, as the degree of relatedness increased, the associative strength was higher, as seen in Table 9.

\section{Discussion}

In Experiment 1, we found evidence that, with increasing age, the distances between prime and target become smaller, as measured by Pathfinder method. Are these distances related to any variable in semantic priming experiments? In semantic priming experiments, associative strength, measured by associative norms (see Table 1), accounts for the differences in semantic priming at short SOAs (Cañas, 1990; Neely, 1991; Nievas et al., 1994). Hence, when the associative strength between primes and targets is different, there are also differences in semantic priming at short SOAs. For example, Cañas (1990) reported differences in semantic priming when the associative strength between primes and targets was $3 \%$ in relation to primes and targets with an associative strength of $40 \%$ at short SOAs (100 ms). Therefore, with low associative strength, reaction time is higher.

Experiment 3 shows that associative strength is positively related to the degree of connection between concepts, measured by Pathfinder ratings, so that, as relatedness between concepts increases, associative strength is higher. Therefore, the ratings used in Pathfinder method may produce differences in semantic priming.

Similarly, results from Experiment 3 suggest that associative strength is negatively related to Pathfinder distances, so that, as distances increase, the associative strength decreases. Therefore, the results from Experiment 3, taken conjointly with those from Experiment 1, revealing differences between young people's and adults' Pathfinder distances, together with the fact that these distances are related to associative strength, may account for the missing semantic priming effect at short SOAs in children.

\section{General Discussion}

In the introduction to this paper, we commented on the semantic priming effect in the word recognition paradigm. One of the effects on semantic priming is that there is no semantic priming in children (Nievas, 1999a, 1999b; Simpson \& Foster, 1986) whereas in adults, it was present at short SOA (Frost \& Bentin, 1992; Nievas, 1999a, 1999b; Simpson \& Burgess, 1985 ). Neely (1991) proposed that the spreading activation theories might explain more data at short SOA. This kind of theory accounts for reaction time results by assuming that concepts in the semantic memory are represented by nodes that are connected, so that when a target is presented, its node is activated. If a target-related prime is presented previously (at short SOA), then reaction time to the target is reduced. This is because the prime node spreads activation to the target node, so that when the target word is presented, its node has already been activated to some extent by the prime word node. Hence, recognition is faster because the amount of activation needed to reach the recognition threshold is lower for the target word node.

Nievas (1999b) proposed some hypotheses to explain why priming is absent in children. One of the hypotheses is based on children's and adults' differences in concept networks. For adults, the prime word node and the target word node may be directly connected, whereas for children, they may be connected via some intermediate nodes. Therefore, when the prime word node spreads activation, the target word node does not receive the activation because of these intermediate nodes and because activation spreading is lower when the destination node is farther away in the network. This connection between nodes could be measured by the Pathfinder method.

Using a method to capture the relations between concepts, we looked for differences in children's and adults' memory structure. We proposed two hypotheses: (a) Children's concept structures may have fewer links than those of adults, in which case, in adults' networks, more activation would accumulate for a node because it would receive more activation from its links; and (b) the distance between prime node and target node may be large in children's networks and small in adults' networks. If so, as distance between the prime and the target node increases, activation received by the target node would be lower.

The first experiment was designed to test whether there were age-related differences between memory structure representations, measured by three dependent variables: number of links, $C$ measures, and distances. Results of the first experiment refute the first proposal. There were no 
differences between the groups in the number of links in the networks, measured by Pathfinder method, except for the Professor Group. With more complex structures $(q=2)$, the Professor Group had fewer links than the other groups. In semantic priming experiments (Nievas, 1999a, 1999b), positive priming was found for adults (Secondary and University) but not for the Primary Group. The results of the first experiment reveal that there are no differences between groups (Primary, Secondary, and University) in the number of links. In other words, the number of links is not a good candidate to account for missing semantic priming in children. In the future, it would be very interesting to design a semantic priming experiment with a Professor Group.

Our second hypothesis stated that the distance between prime node and target node may be large in children's networks and small in adults' networks. Results of the first experiment confirmed this hypothesis. As age increases, Pathfinder distances are shorter with less complex structures $(q=n-1)$. The third experiment revealed the relation between distances, measured by Pathfinder method, and associative strength. In the semantic priming experiments, associative strength was measured by the associative norms frequently used by university students. However, taking our results into account, in order to carry out a semantic priming experiment with children (Primary Group), an associative norm for children should be developed because the distances between children and adults are different. Associative strength may account for missing semantic priming in children.

Another relevant result revealed differences between younger and older people in their organizational memory representations. $C$ measures are higher as age increases, again with $q=n-1$. This result indicates that semantic memory does not seem to be so static, but rather updates over time.

Lastly, our results also indicate that the PUF effect may play a role in Pathfinder network representations. This might enhance researchers' comprehension of the developmental factor of memory representation.

\section{References}

Anderson, J.R. (1976). Language, memory, and thought. Hillsdale, NJ: Erlbaum.

Anderson, J.R. (1983). The architecture of cognition. Cambridge, MA: Harvard University Press.

Besner, D., \& Humphreys, G.W. (1991). Basic processes in word recognition and identification: An overview. In D. Besner \& G. Humphreys (Eds.), Basic processes in reading: Visual word recognition (pp. 1-9). Hillsdale, NJ: Erlbaum.

Cañas, J.J. (1990). Associative strength effects in the lexical decision task. The Quarterly Journal of Experimental Psychology, 42A, 121-145.

Collins, A.M., \& Loftus, E.F. (1975). A spreading-activation theory of semantic processing. Psychological Review, 82, 407-428.
Collins, A.M., \& Quillian, M. R. (1969). Retrieval time from semantic memory. Journal of Verbal Learning and Verbal Behaviour, 8, 240-247.

Frost, R., \& Bentin, S. (1992). Processing phonological and semantic ambiguity: Evidence from semantics priming at different SOAs. Journal of Experimental Psychology: Learning, Memory and Cognition, 18, 58-68.

Glanzer, M., \& Bowles, N. (1976). Analysis of the word-frequency effect in recognition memory. Journal of Experimental Psychology: Human Learning and Memory, 2, 21-31.

Goldberger, A.L., Rigney, D.R., Mietus, J., Antman, E.M., \& Greenwald, S. (1988). Nonlinear dynamics in sudden cardiac death syndrome: Heartrate oscillations and bifurcations. Experientia, 44, 983-987.

Goldberger, A.L., Rigney, D.R., \& West, B.J. (1990). Caos y fractales en la fisiología humana. In A. Fernández-Rañada (Ed.), Orden y caos (pp. 108-116). Barcelona: Prensa Científica.

Goldberger, A.L. \& West, B.J. (1987). Fractals in physiology and medicine. Yale Journal of Biology and Medicine, 60, 421-435.

Goldsmith, T.E., Johnson, P.J., \& Acton, W.H. (1991). Assessing structural knowledge. Journal of Educational Psychology, 83, 88-96.

Gonzalvo, P., Cañas, J.J., \& Bajo, M.T. (1994). Structural representations in knowledge adquisition. Journal of Educational Psychology, 86, 601-616.

Juilland, A., \& Chang-Rodríguez, E. (1964). Frequency dictionary of Spanish words. The Hague, Netherlands: Mouton.

MacKay, D.G. (1987). The organization of perception and action: A theory for language and other cognitive skills. New York: Springer-Verlag.

MacKay, D.G. (1990). Perception, action and awareness: A threebody problem. In O. Newmann \& W. Prinz (Eds.), Relationships between perception and action (pp. 269-303). Berlin: Springer-Verlag.

Meyer, D.E., \& Schvaneveldt, R.W. (1971). Facilitation in recognizing pairs of words: Evidence of a dependence between retrieval operations. Journal of Experimental Psychology, 90, 227-234.

Neely, J.H. (1977). Semantic priming and retrieval from lexical memory: Roles of inhibitionless spreading activation and limited-capacity attention. Journal of Experimental Psychology: General, 106, 226-254.

Neely, J.H. (1991). Semantic priming effects in visual word recognition: A selective review of current findings and theories. In D. Besner \& G.W. Humphreys (Eds.), Basic processes in reading: Visual word recognition (pp. 264-336). Hillsdale, NJ: Erlbaum.

Nievas, F. (1999a, September). A transversal study about meaning access processes for homographs. Poster presented at the IXth European Conference on Developmental Psychology. Spetses, Greece.

Nievas, F. (1999b). Estudio transversal sobre el acceso a los significados de palabras homógrafas. Unpublished doctoral dissertation, University of Almería, Spain.

Nievas, F., \& Cañas, J. J. (1993). Asociados de una base de homógrafos. Psicológica, 14, 269-279. 
Nievas, F., Cañas, J.J., \& Bajo, M.T. (1994, September). Lexical processing of ambiguous words: Dominance or associative strength? Poster presented at VII Conference of European Society of Cognitive Psychology (ESCOP). Lisbon, Portugal.

Nievas, F., \& Marí-Beffa, P. (2002). Negative priming from the non selected meaning of the homograph. British Journal of Psychology, 93, 47-66.

Posner, M.I., \& Snyder, C.R.R. (1975). Attention and cognitive control. In R.L. Solso (Ed.), Information processing and cognition: The Loyola Symposium. Hillsdale, NJ: Erlbaum.

Reder, L., Anderson, J., \& Bjork, R. A. (1974). A semantic interpretation of encoding specificity. Journal of Experimental Psychology, 102, 648-656.

Rumelhart, D.E., \& Norman, D.A. (1981). Accretion, tuning and restructuring: Three modes of Learning. In J. W. Cotton \& R. Klatzky (Eds.), Semantic factors in cognition (pp. 37-54). Hillsdale, NJ: Erlbaum.

Schnorr, J.A., \& Atkinson, R.C. (1970). Study position and item difficulty in the short- and long-term retention of paired associated learned by imagery. Journal of Verbal Learning and Verbal Behavior, 9, 614-622.

Schnotz, W., Vosniadou, S., \& Carretero, M. (Eds.) (1999). New perspectives on conceptual change. Amsterdam: Pergamon.

Schvaneveldt, R.W. (Ed.) (1990). Pathfinder associative networks: Studies in knowledge organization. Norwood, NJ: Ablex.

Schvaneveldt, R.W., Ackerman, B.P., \& Semlear, T. (1977). The effects of semantic context on children's word recognition. Child Development, 48, 612-616.

Schvaneveldt, R.W., Dearholt, D.W., \& Durso, F.T. (1988). Graph theoretic foundations of Pathfinder networks. Computers and Mathematics with Applications, 15, 337-345.

Schvaneveldt, R.W., Durso, F.T., \& Dearholt, D.W. (1989). Network structures in proximity data. In G.H. Bower (Ed.), The psychology of learning and motivation (Vol. 24, pp. 249-284). New York: Academic Press.
Schwantes, F.M. (1981). Locus of the context in children's word recognition. Child Development, 52, 895-903.

Schwantes, F.M., Boesl, S.L., \& Ritz, E.G. (1980). Children's use of context in word recognition: A psycholinguistic guessing game. Child Development, 51, 730-736.

Simpson, G.B., \& Burgess, C. (1985). Activation and selection processes in the recognition of ambiguous words. Journal of Experimental Psychology: Human Perception and Performance, 22, 147-154.

Simpson, G.B., \& Foster, M.R. (1986). Lexical ambiguity and children's word recognition. Developmental Psychology, 22, 147-154.

Simpson, G.B., Krueger, M.A. Kang, H., \& Elofson, A.C. (1994). Sentence context and meaning frequency effects in children's processing of ambiguous words. Journal of Research in Reading, 17, 62-72.

Simpson, G.B., \& Lorsbach, T.C. (1983). The development of automatic and conscious components of contextual facilitation. Child Development, 54, 760-772.

Stanovich, K.E., West, R.F., \& Feeman, D.J. (1981). A longitudinal study of sentence context effects in second-grade children: Test of an interactive-compensatory model. Journal of Experimental Child Psychology, 32, 185-199.

Taft, M. (1991). Reading and the mental lexicon. Hove, UK: Erlbaum.

Vega, M., Carreiras, M., Gutiérrez-Calvo, M., \& Alonso-Quecuty, M.L. (1990). Lectura y comprensión. Una perspectiva cognitiva. Madrid: Alianza Psicología.

West, B.J., \& Goldberger, A.L. (1987). Physiology in fractal dimensions. American Scientist, 75, 354-365.

West, R.F., \& Stanovich, K.E. (1978). Automatic contextual facilitation in readers of three ages. Child Development, 49, 717-727.

Received July 8, 2002

Revision received November 6, 2002 Accepted December 20, 2002 


\section{APPENDIX A}

Meanings of the Spanish words used in Figure 1.

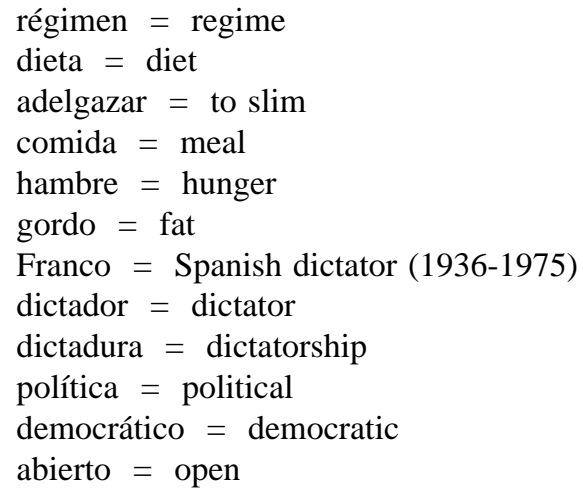




\section{APPENDIX B}

Homographs used in first and second experiment. $\mathbf{D}$ was the dominant associate used and $\mathbf{S}$ the subordinate. The associative strengths for dominant associates were $(M=8.0, S D=3.5)$ and $(M=8.6, S D=5.0)$ for low and medium printed usage frequency, respectively. The associative strengths for subordinate associates were $(M=7.9, S D=1.8)$ and $(M=8.4, S D=4.4)$ for low and medium printed usage frequency, respectively.

Low Printed Usage Frequency:

abonar = to pay; to credit; to fertilize

asta $=$ shaft; wooden stick

apéndice = annex, appendix; blind gut; tail piece

cola = tail; queue; a plant or herb; glue

mango = haft, handle; mango

Medium Printed Usage Frequency:

apuntar = to aim; to write down

corredor* $=$ runner; broker; hall

grano = grain; kernel; pimple; seed

régimen $=$ regime (political, diet)

segundo $=$ second (time, order)
D

S

dar (to give)

cuerno (horn)

índice (index)

pegar (to stick)

asa (haft, handle)

anotar (to annotate)

puertas (doors)

espinilla (blackhead; shin)

dieta (diet)

tercero (third) campo (country)

bandera (flag)

dolor (ache, pain)

rabo (tail)

ropa (clothes)

señalar (to point out)

apuesta (bet)

trigo (wheat)

dictadura (dictatorship)

hora (hour)

* Not used in the Nievas (1999a; 1999b) study. 